K. Moriyasu

Nagoya Math. J.

Vol. 123 (1991), 91-102

\title{
THE TOPOLOGICAL STABILITY OF DIFFEOMORPHISMS
}

\author{
KAZUMINE MORIYASU
}

\section{§. Introduction}

The present paper is concerned with the stability of diffeomorphisms of $C^{\infty}$ closed manifolds. Let $M$ be a $C^{\infty}$ closed manifold and $\operatorname{Diff}^{r}(M)$ be the space of $C^{r}$ diffeomorphisms of $M$ endowed with the $C^{r}$ topology (in this paper we deal with only the case $r=0$ or 1 ). Let us define

$$
\mathscr{F}(M)=\left\{\begin{array}{l|l}
f \in \operatorname{Diff}^{1}(M) & \begin{array}{l}
\text { there exists a } C^{1} \text { neighborhood } \mathscr{U}(f) \text { of } \\
f \text { such that all periodic points of every } \\
g \in \mathscr{U}(f) \text { are hyperbolic }
\end{array}
\end{array} .\right.
$$

Then every $C^{1}$ structurally stable and $\Omega$-stable diffeomorphism belongs to $\mathscr{F}(M)$ (see [3]). In light of this result Mañe solved in [5] the $C^{1}$ Structural Stability Conjecture by Palis and Smale. After that Palis [9] obtained, in proving that every diffeomorphism belonging to $\mathscr{F}(M)$ is approximated by Axiom $A$ diffeomorphisms with no cycle, the $C^{1} \Omega$-Stability Conjecture. Recently Aoki [2] proved that every diffeomorphism belonging to $\mathscr{F}(M)$ is Axiom $A$ diffeomorphisms with no cycle (a conjecture by Palis and Mañé). For the topological stability Walters [14] proved that every Anosov diffeomorphism is topologically stable. In [7] Nitecki showed that every Axiom $A$ diffeomorphism having strong transversality is topologically stable, and that every Axiom $A$ diffeomorphism having no cycle is $\Omega$ topologically stable.

Thus it will be natural to ask whether topologically stable diffeomorphisms belonging to $\operatorname{Diff}^{1}(M)$ satisfy Axiom $A$ and strong transversality.

Let $f \in \operatorname{Diff}^{1}(M)$. Then $f: M \rightarrow M$ is topologically stable if and only if given $\varepsilon>0$ there exists $\delta>0$ such that for any $g \in \operatorname{Diff}^{\circ}(M)$ with $d(f, g)<\delta$ there exists a continuous map $h: M \rightarrow M$ satisfying $h \circ g=f \circ h$ and $d(h, \mathrm{id})<\varepsilon$ (where id is the identity). Note that if $\varepsilon$ is sufficiently small then the above continuous map $h$ is surjective since $h$ is homotopic to id. We denote by $\Omega(f)$ the set of nonwandering points of $f$. A diffeo-

Received July 20, 1990. 
morphism $f$ is $\Omega$-topologically stable if and only if given $\varepsilon>0$ there exists $\delta>0$ such that for any $g \in \operatorname{Diff}^{0}(M)$ with $d(f, g)<\delta$ such that there exists a continuous map $h: \Omega(g) \rightarrow \Omega(f)(h(\Omega(g)) \subset \Omega(f))$ satisfying $h \circ g=f \circ h$ on $\Omega(g)$ and $d(h(x), x)<\varepsilon$ for all $x \in \Omega(g)$.

A sequence $\left\{x_{i} \mid i \in(a, b)\right\}(-\infty \leq a<b \leq \infty)$ of points is called a $\delta$ pseudo orbit for $f$ if $d\left(f\left(x_{i}\right), x_{i+1}\right)<\delta$ for $i \in(a, b-1)$. Given $\varepsilon>0$ a pseudo orbit $\left\{x_{i}\right\}$ is said to be $\varepsilon$-traced by a point $x \in M$ if $d\left(f^{i}(x), x_{i}\right)<\varepsilon$ for $i \in(a, b)$. We say that $f$ has the pseudo orbit tracing property (abbrev. POTP) if for $\varepsilon>0$ there is $\delta>0$ such that every $\delta$-pseudo orbit for $f$ can be $\varepsilon$-traced by some point of $M$.

For compact spaces the notions stated above are independent of the compatible metric used. It is known that if $f: M \rightarrow M$ is topologically stable then $f$ has POTP and all the periodic points of $f$ are dense in $\Omega(f)$ (see [6], [15]), and that if $f: M \rightarrow M$ has POTP then so is $f_{\mid \Omega(f)}: \Omega(f)$ $\rightarrow \Omega(f)$ (see [1]).

To mention precisely our aim let us define the subsets of $\operatorname{Diff}^{1}(M)$ as

$\operatorname{AxS}(M)=\{f \mid f$ satisfies Axiom $A$ and strong transversality $\}$,

$\operatorname{AxN}(M)=\{f \mid f$ satisfies Axiom $A$ and no cycle $\}$,

$\operatorname{POTP}(M)=\operatorname{int}\{f \mid f$ has $\operatorname{POTP}\}$,

$\Omega$-POTP $(M)=\operatorname{int}\left\{f \mid f_{\mid \Omega(f)}\right.$ has $\left.\operatorname{POTP}\right\}$,

$\mathrm{TS}(M)=\operatorname{int}\{f \mid f$ is topologically stable $\}$,

$\Omega$-TS $(M)=\operatorname{int}\{f \mid f$ is $\Omega$-topologically stable $\}$.

Here int $E$ denotes the interior of $E$. Among these sets exist the following

$$
\begin{array}{ll}
\operatorname{POTP}(M) \subset \Omega-\operatorname{POTP}(M)([1]), & \operatorname{TS}(M) \subset \Omega-\mathrm{TS}(M), \\
\operatorname{TS}(M) \subset \operatorname{POTP}(M)([6] \text { or [15]), } & \operatorname{AxS}(M) \subset \operatorname{TS}(M)([7] \text { or [12]), } \\
\operatorname{AxN}(M) \subset \Omega-\operatorname{TS}(M)([7]), & \operatorname{AxN}(M)=\mathscr{F}(M)([2]) .
\end{array}
$$

For the question mentioned above we shall show the following

THEOREM 1. Under the above notations, the following holds.

(1) $\Omega$-TS $(M)=\mathscr{F}(M)$,

(2) $\operatorname{TS}(M)=\operatorname{AxS}(M)$.

By Theorem 1 the following is concluded.

$$
\Omega-\mathrm{TS}(M)=\operatorname{AxN}(M)=\mathscr{F}(M) \subset \mathrm{TS}(M)=\operatorname{AxS}(M) .
$$

We have the following theorem as an easy conclusion of Theorem 1 . 
Theorem 2. Let $f \in \operatorname{POTP}(M)$. If $\operatorname{dim} W^{s}(x, f)=0$ or $\operatorname{dim} M$ or $\operatorname{dim} M$ -1 for $x \in M$, then $f$ belongs to $\operatorname{AxS}(M)$.

The proof of Theorem 2 will be given in $\S 5$.

The conclusions of Theorem 1 will be obtained in proving the following three propositions.

Proposition 1. $\Omega$-POTP $(M) \subset \mathscr{F}(M)$.

The proof will be based on the techniques of the proof of Theorem 1 of Franks [3].

If we establish Proposition 1 , then we have that $\Omega$-POTP $(M)=\mathscr{F}(M)$ by the fact mentioned above.

Proposition 2. $\Omega$-TS $(M) \subset \mathscr{F}(M)$.

For the proof we need the methods in [6] or [15], in which it is proved that topological stability implies POTP, and the facts used in the proof of Proposition 1.

Since Proposition 2 shows $\Omega$-TS $(M)=\mathscr{F}(M)$, (1) of Theorem 1 is concluded.

Proposition 3. $\operatorname{TS}(M) \subset \operatorname{AxS}(M)$.

A result that Axiom A diffeomorphisms satisfying structural stability have strong tansversality was proved in Robinson [11]. However every diffeomorphism dealt with in Proposition 3 is Axiom $A$ and topologically stable. Thus it does not follow from Robinson's result that the diffeomorphism satisfies strong transversality.

Proposition 3 ensures that $\operatorname{TS}(M)=\operatorname{AxS}(M)$ and therefore (2) of Theorem 1 is concluded.

\section{§ 2. Proof of Proposition 1}

Let $P(f)$ denote the set of periodic points of $f \in \Omega$-POTP $(M)$. If $p \in P(f)$ with the prime period $k$, then $T_{p} M$ splits into the direct sum $T_{p} M=E^{u}(p) \oplus E^{s}(p) \oplus E^{c}(p)$ where $E^{u}(p), \quad E^{s}(p)$ and $E^{c}(p)$ are $D_{p} f^{k}$ invariant subspaces corresponding to the absolute values of the eigenvalues of $D_{p} f^{k}$ with greater than one, less than one and equal to one.

To obtain Proposition 1 it suffices to prove that each $p \in P(f)$ is hyperbolic: i.e. $E^{c}(p)=\{0\}$. On the contrary suppose that $p \in P(f)$ is non-hyperbolic and let $k>0$ be the prime period of $p$. Then, for every $\varepsilon>0$ there exists a linear automorphism $\mathcal{O}: T_{p} M \rightarrow T_{p} M$ such that 

( ( i ) $\|\mathcal{O}\| \leq \varepsilon$,
(ii) $\mathcal{O}\left(E^{\sigma}(p)\right)=E^{\sigma}(p)$ for $\sigma=s, u, c$,
(iii) all eigenvalues of $\mathcal{O} \circ D_{p} f^{k} \mid E^{c}(p)$ are of a root of unity.

Making use of the following Franks's lemma, we can find $\delta_{0}>0$ and a diffeomorphism $g \in \Omega$-POTP $(M)$ such that

$$
\left\{\begin{array}{c}
\text { (i) } B_{4 \delta_{0}}\left(f^{i}(p)\right) \cap B_{4 \delta_{0}}\left(f^{j}(p)\right)=\varnothing \text { for } 0 \leq i \neq j \leq k-1, \\
\text { (ii) } g(x)=f(x) \text { for } x \in\left\{p, f(p), \cdots, f^{k-1}(p)\right\} \cup\left\{M-\bigcup_{i=0}^{k-1} B_{4 \delta_{0}}\left(f^{i}(p)\right)\right\}, \\
\text { (iii) } g(x)=\exp _{f^{i+1}(p)} \circ D_{f^{i}(p)} f \circ \exp _{f^{i}(p)}^{-1}(x) \\
\text { for } x \in B_{\delta_{0}}\left(f^{i}(p)\right)(0 \leq i \leq k-2), \\
\text { (iv) } g(x)=\exp _{p} \circ \mathcal{O} \circ D_{f^{k-1}(p)} f \circ \exp _{f^{k-1}(p)}^{-1} \text { for } x \in B_{\delta_{0}}\left(f^{k-1}(p)\right) .
\end{array}\right.
$$

Franks's lemma. For $f \in \operatorname{Diff}^{1}(M)$ let $F$ be a finite set of distinct points in $M$. If $\varepsilon>0$ is sufficiently small and $G_{x}: T_{x} M \rightarrow T_{f(x)} M$ is an isomorphism such that $\left\|G_{x}-D_{x} f\right\|<\varepsilon / 10(x \in F)$, then there exist $\delta>0$ and a diffeomorphism $\mathrm{g}: M \rightarrow M, \varepsilon$ close to $f$ in the $C^{1}$ topology, such that $B_{4 \delta}(x) \cap B_{4 \delta}(y)$ $=\varnothing$ for $x, y \in F$ with $x \neq y$ and $g(z)=\exp _{f(x)} \circ G_{x} \circ \exp _{x}^{-1}(z)$ if $z \in B_{\delta}(x)$ and $g(z)=f(z)$ if $z \notin B_{4 \delta}(x)(x \in F)$.

Define $G=\mathcal{O} \circ D_{p} f^{k}$. Then there exists $m>0$ such that $G_{\mid E^{c}(p)}^{m}$ is the identity by (2.1), and $\delta_{1}>0$ such that

$$
\left.g^{m k}\right|_{\exp _{p} T_{p} M\left(\hat{\delta}_{1}\right)}=\exp _{p} \circ G^{m} \circ \exp _{p}^{-1} \quad(\text { by }(2.2))
$$

where $T_{p} M\left(\delta_{1}\right)=\left\{v \in T_{p} M \mid\|v\| \leq \delta_{1}\right\}$. Put $E^{c}\left(p, \delta_{1}\right)=E^{c}(p) \cap T_{p} M\left(\delta_{1}\right)$, then it is clear that

$$
\left.g^{m k}\right|_{\exp _{p} E^{c}\left(p, \delta_{1}\right)}=\left.\mathrm{id}\right|_{\exp _{p} E^{c}\left(p, \tilde{o}_{1}\right)} .
$$

Since $g \in \Omega$-POTP $(M)$, we see that $g_{\mid \Omega(g)}^{m k}$ has POTP. Then, for $0<\varepsilon<\delta_{1} / 4$ there exists $0<\delta<\varepsilon$ such that every $\delta$-pseudo orbit is $\varepsilon$-traced by some point in $\Omega(g)$. Now take and fix $y \in \exp _{p} E^{c}\left(p, \delta_{1}\right)$ with $d(p, y)=\frac{3}{4} \delta_{1}$. From (2.4) we can construct a cyclic $\delta$-pseudo orbit $\left\{x_{i}\right\}$ of $g^{m k}$ satisfying

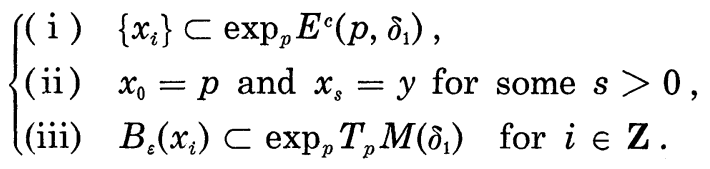

For the pseudo orbit $\left\{x_{i}\right\}$ there is $z \in \Omega(g)$ such that $d\left(g^{m k i}(z), x_{i}\right)<\varepsilon$ for $i \in \mathbf{Z}$ as explained above. By (2.5) (iii) we have $\exp _{p}^{-1} \circ g^{m k i}(z) \in T_{p} M\left(\delta_{1}\right)$ and letting $u=\exp _{p}^{-1} z,\left\|G^{m i}(u)\right\|=\left\|\exp _{p}^{-1} \circ g^{n k i}(z)\right\| \leq \delta_{1}$ for $i \in \mathbf{Z}$. Thus 
$u \in E^{c}(p)$ and so $z \in \exp _{p} E^{c}\left(p, \delta_{1}\right)$. From (2.4) we have that $d(p, z) \geq d\left(p, x_{s}\right)$ $-d\left(x_{s}, z\right)=d(p, y)-d\left(x_{s}, g^{m k s}(z)\right) \geq \frac{3}{4} \delta_{1}-\varepsilon>\frac{1}{2} \delta_{1}>\varepsilon$, which shows a contradiction.

\section{§ 3. Proof of Proposition 2}

Let $f \in \Omega-T S(M)$. For $\varepsilon>0$ there exists $\delta>0$ such that for $g \in \operatorname{Diff}^{0}(M)$, $d\left(f(x), g\left(x_{s}\right)\right) \leq \delta(x \in M)$ implies that there exists a continuous map $h: \Omega(g)$ $\rightarrow \Omega(f)$ satisfying $h \circ g=f \circ h$ and $d(h(x), x) \leq \varepsilon$ for $x \in \Omega(g)$. Note that $g$ does not belong to $\Omega$-TS $(M)$.

The proof is divided into two the cases $\operatorname{dim} M=1$ and $\operatorname{dim} M \geq 2$. For the case $\operatorname{dim} M=1$ we know that the set of all Morse-Smale diffeomorphisms is open dense in $\operatorname{Diff}^{1}(M)$. Choose a Morse-Smale diffeomorphism as the diffeomorphism $g$. Then, it is easily checked that $h(P(g))=P(f)$. Thus $\sharp P(f) \leq \# P(g)<\infty$, which implies that $f_{\mid \vec{P}(f)}$ has POTP. Therefore $f \in \mathscr{F}(M)$ by the same proof as Proposition 1.

For the case $\operatorname{dim} M \geq 2$, we prove directly that $f \in \mathscr{F}(M)$. To do this it suffices to show that every $x \in P(f)$ is hyperbolic. Suppose that $p \in P(f)$ is non-hyperbolic and let $k>0$ be a prime period of $p$. As in the proof of Proposition 1, for $\varepsilon>0$ take a linear automorphism $\mathcal{O}: T_{p} M \rightarrow T_{p} M$ satisfying (2.1) and after that take $\delta_{0}>0$ and $g \in \Omega$-TS $(M)$ satisfying (2.2). Moreover let $m>0$ be a minimal integer such that $G_{\mid E^{c}(p)}^{m}$ is the identity map on $E^{c}(p)$. We put $I_{0}=E^{c}(p)$ when $m=1$. If $m \geq 2$ then we take $v \in E^{c}(p)$ with $\|v\|=1$ such that, letting $I_{0}=\{t v \mid t \geq 0\}$ and $I_{l}=G^{l}\left(I_{0}\right)$ $(0 \leq l \leq m-1)$

$$
\left\{\begin{array}{l}
\text { ( i ) } I_{l} \cap I_{l^{\prime}}=\{0\} \quad\left(0 \leq l \neq l^{\prime} \leq m-1\right), \\
\text { (ii) } G^{m}\left(I_{l}\right)=I_{l} \quad(0 \leq l \leq m-1) .
\end{array}\right.
$$

Let $\delta_{1}>0$ be as in (2.3) and take $y \in \exp _{p}\left(I_{0} \cap T_{p} M\left(\delta_{1}\right)\right)$ with $d(p, y)=\frac{3}{4} \delta_{1}$. Since $g_{\exp _{p} E^{c}\left(p, \delta_{1}\right)}^{m k}$ is the identity on $\exp _{p} E^{c}\left(p, \delta_{1}\right)$ by $(2.4)$, for $0<\varepsilon<\frac{1}{4} \delta_{1}$ and every $\delta>0$ we can find a finite sequence $\left\{x_{i}\right\}_{i=0}^{2 s}$ of $M$ such that

$$
\begin{cases}\text { (i ) } & \left\{x_{i}\right\}_{i=0}^{2 s} \subset \exp _{p}\left\{\left(I_{0} \cap T_{p} M\left(\delta_{1}\right)\right)-\{0\}\right\}, \\ \text { (ii) } & d\left(x_{0}, p\right)<\varepsilon, \\ \text { (iii) } & x_{2 s}=x_{0} \text { and } x_{s}=y, \\ \text { (iv) } & x_{i} \neq x_{j} \text { for } 0 \leq i \neq j \leq 2 s-1, \\ \text { (v) } & d\left(x_{i}, x_{i+1}\right)<\delta \text { for } 0 \leq i \leq 2 s-1, \\ \text { (vi) } & B_{\varepsilon}\left(x_{i}\right) \in \exp _{p} T_{p} M\left(\delta_{1}\right) \text { for } 0 \leq i \leq 2 s .\end{cases}
$$


Now define $p_{m k i}=x_{i}$ and $q_{m k i}=x_{i+1}$ and $p_{m k i+j}=q_{m k i+j}=g^{j}\left(x_{i+1}\right)$ for $0 \leq i \leq 2 s-1$ and $1 \leq j \leq m k-1$. Then we have that $d\left(p_{n}, q_{n}\right)<\delta$, $p_{n} \neq p_{n^{\prime}}$ and $q_{n} \neq q_{n^{\prime}}$ for $0 \leq n \neq n^{\prime} \leq 2 s m k-1$. Thus, by Lemma 13 of Nitecki and Shub [8] we have that there exists $\varphi \in \operatorname{Diff}^{1}(M)$ such that $d(\varphi(x), x)<2 \pi \delta$ for $x \in M$ and $\varphi\left(p_{n}\right)=q_{n}$ for $0 \leq n \leq 2 s m k-1$. Define $\tilde{g}=g \circ \varphi$. Since $\delta$ is arbitrary, we can take $\tilde{\mathrm{g}}$ such that $\tilde{\mathrm{g}}$ is small $C^{0}$ near to $g$. Thus there exists a continuous map $h: \Omega(\tilde{\mathrm{g}}) \rightarrow \Omega(g)$ satisfying $h \circ \tilde{\mathrm{g}}=\mathrm{g} \circ h$ and $d(h(x), x)<\varepsilon$ for $x \in \Omega(\tilde{\mathrm{g}})$. Moreover $\tilde{\mathrm{g}}^{2 s m k}\left(x_{0}\right)=x_{0}$. Thus $d\left(p, h\left(x_{0}\right)\right) \leq d\left(p, x_{0}\right)+d\left(x_{0}, h\left(x_{0}\right)\right) \leq 2 \varepsilon$ and

$$
\begin{aligned}
d\left(y, g^{s m k}\left(h\left(x_{0}\right)\right)\right) & =d\left(y, h\left(\tilde{g}^{s m k}\left(x_{0}\right)\right)\right) \\
& =d\left(y, h\left(x_{s}\right)\right) \leq d\left(y, x_{s}\right)+d\left(x_{s}, h\left(x_{s}\right)\right) \leq \varepsilon .
\end{aligned}
$$

Therefore we have $\frac{3}{4} \delta_{1}=d(p, y) \leq d\left(p, h\left(x_{0}\right)\right)+d\left(g^{s m k}\left(h\left(x_{0}\right)\right), y\right) \leq 3 \varepsilon<\frac{3}{4} \delta_{1}$, since $g^{s m k}\left(h\left(x_{0}\right)\right)=h\left(x_{0}\right)$. We arrived at a contradiction.

\section{§4. Proof of Proposition 3}

Since $T S(M) \subset \mathscr{F}(M)$ by Propositions 1 and 2 , it is clear that $f \epsilon$ $T S(M)$ satisfies Axiom $A$ and no cycle. Thus $f$ is $\Omega$-stable. On the other hand, since $f$ is topologically stable, for $\varepsilon>0$ small enough we can find a small neighborhood $\mathscr{U}(f)$ of $f$ in $\operatorname{Diff}^{1}(M)$ such that for $g \in \mathscr{U}(f)$ there exists a continuous surjection $h: M \rightarrow M$ such that $h \circ g(x)=f \circ h(x)$ and $d(h(x), x)<\varepsilon$ for all $x \in M$ and moreover $h_{\mid \Omega(g)}: \Omega(g) \rightarrow \Omega(f)$ is bijective.

Thus we have that for $x \in M$

$$
h^{-1} W^{\sigma}(h(x), f)=W^{\sigma}(x, g) \quad(\sigma=s, u)
$$

where

$$
\begin{aligned}
& W^{s}(x, g)=\left\{y \in M \mid d\left(g^{n}(x), g^{n}(y)\right) \rightarrow 0 \text { as } n \rightarrow \infty\right\}, \\
& W^{u}(x, g)=\left\{y \in M \mid d\left(g^{-n}(x), g^{-n}(y)\right) \rightarrow 0 \text { as } n \rightarrow \infty\right\} .
\end{aligned}
$$

Indeed, (4.1) is checked as follows. Since $f \in \mathscr{F}(M)$ and $\mathscr{U}(f)$ is a sufficiently small neighborhood, we can take it as $\mathscr{U}(f) \subset \mathscr{F}(M)$. Thus

$$
M=\bigcup_{x \in \Omega(g)} W^{\sigma}(x, g) \quad \text { for } g \in \mathscr{U}(f)(\sigma=s, u) .
$$

Since $h W^{\sigma}(x, g) \subset W^{\sigma}(h(x), f)$ for $x \in M$, we have $W^{\sigma}(x, g) \subset h^{-1} \circ h W^{\sigma}(x, g)$ $\subset h^{-1} W^{o}(h(x), f)$. To obtain (4.1) suppose that $W^{\sigma}(x, g) \neq h^{-1} W^{\sigma}(h(x), f)$. Then $y \notin W^{\circ}(x, g)$ and $h(y) \in W^{\circ}(h(x), f)$ for some $y \in M$. By (4.2) there exist $x^{\prime}, y^{\prime} \in \Omega(g)$ such that $W^{\sigma}\left(x^{\prime}, g\right)=W^{\sigma}(x, g)$ and $W^{o}\left(y^{\prime}, g\right)=W^{\sigma}(y, g)$. Then 
we have

$$
h(y) \in W^{\sigma}(h(x), f) \cap W^{\sigma}(h(y), f)=W^{o}\left(h\left(x^{\prime}\right), f\right) \cap W^{\sigma}\left(h\left(y^{\prime}\right), f\right)
$$

and so $W^{\sigma}\left(h\left(x^{\prime}\right), f\right)=W^{\sigma}\left(h\left(y^{\prime}\right), f\right)$. For $\sigma=s$ we have $d\left(h \circ g^{n}\left(x^{\prime}\right), h \circ g^{n}\left(y^{\prime}\right)\right)$ $=d\left(f^{n} \circ h\left(x^{\prime}\right), f^{n} \circ h\left(y^{\prime}\right)\right) \rightarrow 0$ as $n \rightarrow \infty$. Since $h_{\mid \Omega(g)}$ is a homeomorphism, it follows $d\left(g^{n}\left(x^{\prime}\right), g^{n}\left(y^{\prime}\right)\right) \rightarrow 0$ as $n \rightarrow \infty$ and hence $y^{\prime} \in W^{s}\left(x^{\prime}, g\right)=W^{s}(x, g)$. Therefore $y \in W^{s}(x, g)$ which is a contradiction. Similarly we can derive a contradiction for $\sigma=u$.

Next we check that for $x \in M$

$$
\operatorname{dim} W^{s}(x, f)+\operatorname{dim} W^{u}(x, f) \geq \operatorname{dim} M .
$$

Since $h_{\mid \Omega(g)}$ is bijective, for $p, q \in P(f)$ with $W^{s}(p, f) \cap W^{u}(q, f) \neq \varnothing$ there exist $p^{\prime}, q^{\prime} \in P(g)$ satisfying $h\left(p^{\prime}\right)=p$ and $h\left(q^{\prime}\right)=q$. From (4.1) we have

$$
\begin{aligned}
W^{s}\left(p^{\prime}, g\right) \cap W^{u}\left(q^{\prime}, g\right) & =h^{-1}\left[W^{s}\left(h\left(p^{\prime}\right), f\right) \cap W^{u}\left(h\left(q^{\prime}\right), f\right)\right] \\
& =h^{-1}\left[W^{s}(p, f) \cap W^{u}(q, f)\right] \neq \varnothing .
\end{aligned}
$$

Use here the fact that the set of all Kupka-Smale diffeomorphisms is residual in $\operatorname{Diff}^{1}(M)$. Then we can take a Kupka-Smale diffeomorphism as the diffeomorphism $g$. Thus $\operatorname{dim} W^{s}\left(p^{\prime}, g\right)+\operatorname{dim} W^{u}\left(q^{\prime}, g\right) \geq \operatorname{dim} M$. Since $g$ is $C^{1}$ near to $f$, we have that $\operatorname{dim} W^{\sigma}(x, g)=\operatorname{dim} W^{\circ}(h(x), f)$ for $x \in \Omega(g)$ $(\sigma=s, u)$. Therefore (4.3) was obtained for this case.

Since $f$ satisfies Axiom $A$, there exists $\varepsilon>0$ such that $\bigcap_{n \in Z} f^{n}\left(U_{\varepsilon}\left(\Lambda_{i}\right)\right)$ $=\Lambda_{i}$ for each basic set $\Lambda_{i}$ of $\Omega(f)$. Since topological stability derives POTP, for the number $\varepsilon>0$ let $\delta>0$ be a number satisfying properties in the definition of POTP. Since $M=\cup_{y \in \Omega(f)} W^{\sigma}(y, f)$ for $\sigma=s$, $u$, for $x \in M$ there exist $y_{i} \in \Lambda_{i}$ and $y_{j} \in \Lambda_{j}$ such that $x \in W^{s}\left(y_{i}, f\right) \cap W^{u}\left(y_{j}, f\right)$. Take $m>0$ so large that $d\left(f^{m}(x), f^{m}\left(y_{i}\right)\right)<\delta$ and $d\left(f^{-m}(x), f^{-m}\left(y_{j}\right)\right)<\delta$. Since $\Lambda_{k} \cap P(f)$ is dense in $\Lambda_{k}$ for each basic set $\Lambda_{k}$, we can choose periodic points $p_{i} \in \Lambda_{i}$ and $p_{j} \in \Lambda_{j}$ satisfying $d\left(f^{m}(x), p_{i}\right) \leq \delta$ and $d\left(f^{-m}(x), p_{j}\right) \leq \delta$. Then a $\delta$-pseudo orbit $\mathcal{O}=\left\{\cdots, f^{-2}\left(p_{j}\right), f^{-1}\left(p_{j}\right), f^{-m}(x), \cdots, x, \cdots, f^{m-1}(x), p_{i}\right.$, $\left.f\left(p_{i}\right), \cdots\right\}$ is $\varepsilon$-traced by a point $z$ in $M$. Obviously $z \in W^{s}\left(f^{-m}\left(p_{i}\right), f\right) \cap$ $W^{u}\left(f^{m}\left(p_{j}\right), f\right)$, and hence $\operatorname{dim} W^{s}\left(f^{-m}\left(p_{i}\right), f\right)+\operatorname{dim} W^{u}\left(f^{n}\left(p_{j}\right), f\right) \geq \operatorname{dim} M$ as above. Therefore we have

$$
\begin{aligned}
\operatorname{dim} W^{s}(x, f)+\operatorname{dim} W^{u}(x, f) & =\operatorname{dim} W^{s}\left(p_{i}, f\right)+\operatorname{dim} W^{u}\left(p_{j}, f\right) \\
& =\operatorname{dim} W^{s}\left(f^{-m}\left(p_{i}\right), f\right)+\operatorname{dim} W^{u}\left(f^{m}\left(p_{j}\right), f\right) \\
& \geq \operatorname{dim} M .
\end{aligned}
$$

We now are ready to prove Proposition 3. 
For $x \in M-\Omega(f)$ it suffices to prove that $W^{s}(x, f)$ and $W^{u}(x, f)$ meet transversally. Since $M=\cup_{y \in \Omega(f)} W^{o}(y, f)$ for $\sigma=s$, $u$, there exist $y_{1}, y_{2} \in$ $\Omega(f)$ such that

$$
W^{s}(x, f)=W^{s}\left(y_{1}, f\right) \quad \text { and } \quad W^{u}(x, f)=W^{u}\left(y_{2}, f\right) .
$$

We know (cf. see [4]) that there is $\varepsilon_{1}>0$ with $B_{\varepsilon_{1}}(x) \cap B_{\varepsilon_{1}}(\Omega(f))=\varnothing$ such that for $0<\varepsilon<\varepsilon_{1}$ and $y \in \Omega(f)$

$$
\left\{\begin{array}{l}
\text { (i ) } W_{\varepsilon}^{\sigma}(y, f) \text { is a } C^{1} \text {-disk for } \sigma=s, u, \\
\text { (ii) } W^{s}(y, f)=\bigcup_{n \geq 0} f^{-n}\left(W_{\varepsilon}^{s}\left(f^{n}(y), f\right)\right), \\
\text { (iii) } W^{u}(y, f)=\bigcup_{n \geq 0} f^{n}\left(W_{\varepsilon}^{u}\left(f^{-n}(y), f\right)\right) .
\end{array}\right.
$$

Thus, for $0<\varepsilon_{2}<\varepsilon_{1}$ there exist $n_{1}, n_{2}>0$ satisfying

$$
\begin{cases}(\text { i }) & f^{n_{1}}(x) \in \operatorname{int} W_{\varepsilon_{2}}^{s}\left(f^{n_{1}}\left(y_{1}\right), f\right), \\ \text { (ii) } & f^{-n_{2}}(x) \in \operatorname{int} W_{\varepsilon_{2}}^{u}\left(f^{-n_{2}}\left(y_{2}\right), f\right)\end{cases}
$$

where int $W_{\varepsilon_{2}}^{\sigma}(y, f)$ denotes the interior of $W_{\varepsilon_{2}}^{\sigma}(y, f)$ in $W_{\varepsilon_{1}}^{\sigma}(y, f)$, and $\delta_{0}>0$ satisfying

$$
\begin{cases}\text { (i) } & B_{\delta_{0}}\left(f^{n}(x)\right) \cap B_{\delta_{0}}\left(f^{m}(x)\right)=\varnothing \text { for }-n_{2} \leq n \neq m \leq-n_{1}, \\ \text { (ii) } & f^{-1}\left[B_{\delta_{0}}\left(f^{-n_{2}}(x)\right)\right] \cap B_{\delta_{0}}\left(f^{n}(x)\right)=\varnothing \text { for }-n_{2} \leq n \leq n_{1}, \\ \text { (iii) } & f^{m}\left[B_{\delta_{0}}\left(f^{n}(x)\right)\right] \cap B_{\delta_{0}}\left(f^{n}(x)\right)=\varnothing \text { for }-n_{2} \leq n \leq n_{1} \text { and } m \neq 0 .\end{cases}
$$

Denote by $C_{\tilde{o}}^{\sigma}(y, f)$ the connected component of $y$ in $B_{\delta}(y) \cap W^{\sigma}(y, f)$ for $\sigma=s, u$. From (4.4) and (4.5) it follows that there is $0<\delta_{1}<\delta_{0}$ such that for $0<\delta \leq \delta_{1}$

$$
\begin{aligned}
\operatorname{int} W_{\varepsilon_{2}}^{s}\left(f^{n_{1}}\left(y_{1}\right), f\right) \cap B_{\delta}\left(f^{n_{1}}(x)\right)= & W_{\varepsilon_{1}}^{s}\left(f^{n_{1}}\left(y_{1}\right), f\right) \cap B_{\delta}\left(f^{n_{1}}(x)\right) \\
= & C_{\delta}^{s}\left(f^{n_{1}}(x), f\right), \\
\operatorname{int} W_{\varepsilon_{2}}^{u}\left(f^{-n_{2}}\left(y_{2}\right), f\right) \cap B_{\delta}\left(f^{-n_{2}}(x)\right) & =W_{\varepsilon_{1}}^{u}\left(f^{-n_{2}}\left(y_{2}\right), f\right) \cap B_{\delta}\left(f^{-n_{2}}(x)\right) \\
& =C_{\delta}^{u}\left(f^{-n_{2}}(x), f\right) .
\end{aligned}
$$

Let $\mathscr{U}(f)$ be a small neighborhood of $f$ in $\operatorname{TS}(M)$. Given a sufficiently small $0<\delta_{2}<\delta_{1}$ we can construct diffeomorphisms $\varphi_{i}(i=1,2), C^{1}$ near to the identity, such that

$$
\left\{\begin{array}{l}
\varphi_{1}\left(f^{n_{1}}(x)\right)=f^{n_{1}}(x), \\
\varphi_{1}\left(C_{\delta_{1}}^{s}\left(f^{n_{1}}(x), f\right) \cap B_{\delta_{2}}\left(f^{n_{1}}(x)\right)=\exp _{f^{n_{1}(x)}}\left(D f^{n_{1}} E_{1}\right)\left(\delta_{2}\right),\right. \\
\varphi_{1}=\mathrm{id} \text { on } M-B_{\delta_{1}}\left(f^{n_{1}}(x)\right), \\
f \circ \varphi_{1}^{-1} \in \mathscr{U}(f)
\end{array}\right.
$$


where $E_{1}$ denotes the tangent space at $x$ of $W^{s}(x, f)$, and

$$
\left\{\begin{array}{l}
\varphi_{2}\left(f^{-n_{2}}(x)\right)=f^{-n_{2}}(x) \\
\varphi_{2}\left(C_{\delta_{2}}^{u}\left(f^{-n_{2}}(x), f\right) \cap B_{\delta_{2}}\left(f^{-n_{2}}(x)\right)=\exp _{f-n_{2}(x)}\left(D f^{-n_{2}} E_{2}\right)\left(\delta_{2}\right) .\right. \\
\varphi_{2}=\mathrm{id} \text { on } M-B_{\delta_{1}}\left(f^{-n_{2}}(x)\right), \\
\varphi_{2} \circ f \in \mathscr{U}(f)
\end{array}\right.
$$

where $E_{2}=T_{x} W^{u}(x, f)$. In general $\varphi_{1}$ and $\varphi_{2}$ can be constructed as follows. For $y \in \Omega(f)$ let $F_{1}=T_{y} W^{s}(y, f)$ and write $F_{2}=F_{1}^{\perp}$. Since there exists a $C^{1} \operatorname{map} \gamma: F_{1}(\delta) \rightarrow F_{2}$ such that $\operatorname{graph}(\gamma)=\exp _{y}^{-1}\left(C_{\delta}^{s}(y, f)\right)$, we can define a $C^{1}$ embedding $Q: T_{y} M(\delta) \rightarrow T_{y} M$ satisfying $Q(z)=Q\left(z_{1}, z_{2}\right)=\left(z_{1}, z_{2}+\gamma\left(z_{1}\right)\right)$ for $z=\left(z_{1}, z_{2}\right) \in\left(F_{1} \oplus F_{2}\right) \cap T_{y} M(\delta)$. Clearly $D_{0} Q=$ id and so $Q$ is $C^{1}$ near to $\operatorname{id}_{\mid T_{y M(\delta)}}$ when $\delta$ is small enough. As usual define a $C^{\infty}$ bump function $\alpha: \mathbf{R} \rightarrow[0,1]$ such that $\alpha(t)=0$ if $|t| \leq 1, \alpha(t)=1$ if $|t| \geq 2$ and $\left|\alpha^{\prime}(t)\right|<2$. Then, for a sufficiently small $\delta^{\prime}$ with $0<2 \delta^{\prime}<\delta$ we set

$$
\varphi(z)=\left\{\begin{array}{r}
z \text { if } z \notin B_{\delta}(y) \\
\exp _{y}\left\{k \cdot \exp _{y}^{-1} z+(1-k) Q^{-1}\left(\exp _{y}^{-1} z\right)\right\} \text { if } z \in B_{\delta}(y) \\
\text { where } k=\alpha\left(\frac{\left\|\exp _{y}^{-1} z\right\|}{\delta^{\prime}}\right) .
\end{array}\right.
$$

Then $\varphi: M \rightarrow M$ is a diffeomorphism $C^{1}$ near to id such that $\varphi(y)=y$ and $\varphi\left(C_{\dot{\delta}^{\prime}}^{s}(y, f)\right)=F_{1}\left(\delta^{\prime}\right)$.

As the finite set $F$ and the isomorphism $G_{x}$ of Franks's lemma (mentioned above), we set $F=\left\{f^{-n_{2}}(x), f^{-n_{2}+1}(x), \cdots, f^{n_{1}-1}(x)\right\}$ and $G_{f^{n}(x)}=$ $D_{f^{n}(x)} f\left(-n_{2} \leq n \leq n_{1}-1\right)$. Then we see that for $0<\delta_{2}<\delta_{1}$ small enough there is $g_{3} \in \mathscr{U}(f)$ satisfying

$$
\left\{\begin{array}{l}
g_{3}\left(f^{n}(x)\right)=f^{n+1}(x) \text { for }-n_{2} \leq n \leq n_{1}-1, \\
g_{3}=f \text { on } M-\bigcup_{n=-n_{2}}^{n_{1}-1} B_{\delta_{1}}\left(f^{n}(x)\right), \\
g_{3}=\exp _{f^{n+1}(x)} \circ D_{f^{n}(x)} f \circ \exp _{f^{n}(x)}^{-1} \\
\quad \text { on } B_{\delta_{2}}\left(f^{n}(x)\right) \text { for }-n_{2} \leq n \leq n_{1}-1 .
\end{array}\right.
$$

Thus by (4.6) we can define a diffeomorphism $g$ belonging to $\mathscr{U}(f)$ by

$$
g(y)= \begin{cases}f \circ \varphi_{1}^{-1}(y) & \text { if } y \in B_{\delta_{1}}\left(f^{n_{1}}(x)\right) \\ \varphi_{2} \circ f(y) & \text { if } y \in f^{-1}\left(B_{\delta_{1}}\left(f^{-n_{2}}(x)\right)\right) \\ g_{3}(y) & \text { otherwise }\end{cases}
$$

Then it is easily checked that for $\delta_{3}>0$ small enough 


$$
\left\{\begin{aligned}
(\text { i }) \quad g^{-n_{1}}\left(\operatorname{int} W_{\varepsilon_{2}}^{s}\left(g^{n_{1}}\left(y_{1}\right), g\right)\right) \cap B_{\delta_{3}}(x) & =g^{-n_{1}}\left(W_{\varepsilon_{1}}^{s}\left(g^{n_{1}}\left(y_{1}\right), g\right)\right) \cap B_{\delta_{3}}(x) \\
& =\exp _{x}\left(E_{1}\left(\delta_{3}\right)\right) \\
(\text { ii }) \quad g^{n_{2}}\left(\operatorname{int} W_{\varepsilon_{2}}^{u}\left(g^{-n_{2}}\left(y_{2}\right), g\right)\right) \cap B_{\delta_{3}}(x) & =g^{n_{2}}\left(W_{\varepsilon_{1}}^{u}\left(g^{-n_{2}}\left(y_{2}\right), g\right)\right) \cap B_{\delta_{3}}(x) \\
& =\exp _{x}\left(E_{2}\left(\delta_{3}\right)\right)
\end{aligned}\right.
$$

To obtain the conclusion suppose that $W^{s}(x, f)$ is not transversal to $W^{u}(x, f)$. Then $E=E_{1} \cap E_{2}$ is non trivial $(E \neq\{0\})$ by (4.3). Since $g \in \mathscr{U}(f)$ $(\subset \mathrm{TS}(M))$, take $\varepsilon>0$ smaller than $\min \left\{\varepsilon_{1}-\varepsilon_{2}, \delta_{3} / 4\right\}$, and let $\delta>0$ be a number satisfying the definition of the topological stability i.e. for $\tilde{g} \in$ $\operatorname{Diff}^{\circ}(M)$ with $d(g, \tilde{g}) \leq \delta$, there exists a continuous surjection $h: M \rightarrow M$ satisfying $h \circ \tilde{g}=g \circ h$ and $d(h(y), y) \leq \varepsilon$ for all $y \in M$. For $\delta^{\prime}>0$ sufficiently small we can find a homeomorphism $\mathcal{O}: T_{x} M \rightarrow T_{x} M$ with $\mathcal{O}(0)=0$ such that

(i ) $\max \left\{\left\|\mathcal{O}^{-1}(u)-u\right\|: u \in T_{x} M\left(\delta_{3}\right)\right\} \leq \delta^{\prime}$,

(ii) letting $E^{\prime}=\mathcal{O}\left(E_{1}\right) \cap E_{2}$,

(a) $E^{\prime}$ is a non trivial linear subspace, or $E^{\prime}=\{0\}$,

(b) $E^{\prime} \subset E$,

(c) $\operatorname{dim} E^{\prime}<\operatorname{dim} E$.

Put $\tilde{g}=g$ on $M-B_{\delta_{3}}(x)$ and $\tilde{g}=g \circ \exp _{x} \circ \mathcal{O}^{-1} \circ \exp _{x}^{-1}$ on $B_{\delta_{3} / 2}(x)$. Then $\tilde{g} \in \operatorname{Diff}^{0}(M)$ and $d(g, \tilde{g}) \leq \delta$. Thus we have

$$
\tilde{g}^{-n_{1}}\left(W_{s_{1}}^{s}\left(\tilde{g}^{n_{1}}\left(y_{1}\right), \tilde{g}\right)\right) \cap B_{\delta_{3} / 2}(x)=\mathcal{O}\left(E_{1}\right)\left(\delta_{3} / 2\right)
$$

and by (4.7)

$$
\tilde{g}^{-n_{1}}\left(W_{\varepsilon_{1}}^{s}\left(\tilde{g}^{n_{1}}\left(y_{1}\right), \tilde{g}\right)\right) \cap \tilde{g}^{n_{2}}\left(W_{\varepsilon_{1}}^{u}\left(\tilde{g}^{-n_{2}}\left(y_{2}\right), \tilde{g}\right)\right) \cap B_{\delta_{3} / 2}(x)=\exp _{x}\left(E^{\prime}\left(\delta_{3} / 2\right)\right) .
$$

Since $\operatorname{dim} E^{\prime}<\operatorname{dim} E$ by (ii) (c), if $\varepsilon>0$ is sufficiently small, then we can take $z^{\prime} \in \exp _{x}\left(E\left(\delta_{3}\right)\right)$ satisfying $z^{\prime} \notin B_{\varepsilon}\left(\exp _{x} E^{\prime}\left(\delta_{3} / 2\right)\right)$ and $d\left(z^{\prime}, x\right) \leq \delta_{3} / 4$. Let $h: M \rightarrow M$ be a semi-conjugacy found as above for $\tilde{g}$. Then $h(z)=z^{\prime}$ for some $z \in M$. Since $d\left(z, z^{\prime}\right)=d(z, h(z)) \leq \varepsilon$, we have that $z \notin \exp _{x}\left(E^{\prime}\left(\delta_{3} / 2\right)\right)$ and $d(z, x) \leq d\left(z, z^{\prime}\right)+d\left(z^{\prime}, x\right) \leq \varepsilon+\delta_{3} / 4<\varepsilon_{3} / 2$. Thus, by (4.8).

$$
z \notin \tilde{g}^{-n_{1}}\left(W_{\varepsilon_{1}}^{s}\left(\tilde{g}^{n_{1}}\left(y_{1}\right), \tilde{g}\right)\right) \cap \tilde{g}^{n_{2}}\left(W_{\varepsilon_{1}}^{u}\left(\tilde{g}^{-n_{2}}\left(y_{2}\right), \tilde{g}\right)\right) .
$$

If $z$ does not belong to the left hand set of the above relation, then $d\left(\tilde{g}^{n}(z), \tilde{g}^{n}\left(y_{1}\right)\right)=d\left(\tilde{g}^{n}(z), g^{n}\left(y_{1}\right)\right)>\varepsilon_{1}$ for a certain $n$ larger than $n_{1}$ and by (4.7).

$$
h(z) \in \exp _{x}\left(E\left(\delta_{3}\right)\right) \subset \exp _{x}\left(E_{1}\left(\delta_{3}\right)\right)=g^{-n_{1}}\left(\operatorname{int} W_{\varepsilon_{2}}^{s}\left(g^{n_{1}}\left(y_{1}\right), g\right)\right) \cap B_{\delta_{3}}(x) .
$$

Thus $d\left(g^{n}\left(y_{1}\right), g^{n}(h(z))\right) \leq \varepsilon_{2}$ and so 


$$
\begin{aligned}
d\left(h\left(\tilde{g}^{n}(z)\right), \tilde{g}^{n}(z)\right) & =d\left(g^{n}(h(z)), \tilde{g}^{n}(z)\right) \\
& \geq d\left(\tilde{g}^{n}(z), g^{n}\left(y_{1}\right)\right)-d\left(g^{n}\left(y_{1}\right), g^{n}(h(z))\right)>\varepsilon_{1}-\varepsilon_{2}>\varepsilon .
\end{aligned}
$$

This is inconsistent with the property of $h$. For the case

$$
z \notin \tilde{g}^{n_{2}}\left(W_{\varepsilon_{1}}^{u}\left(\tilde{g}^{-n_{2}}\left(y_{2}\right), \tilde{g}\right)\right)
$$

we obtain a contradiction by the same way. Therefore $W^{s}(x, f)$ is transversal to $W^{u}(x, f)$ for all $x \in M$. The proof of Proposition 3 is complete.

\section{§5. Proof of Theorem 2}

As in the proof of Proposition 3 , we can construct $g \in \operatorname{POTP}(M)$ satisfying (4.7). Now assume that $\operatorname{dim} W^{s}(x, f)=\operatorname{dim} M-1$ and $W^{s}(x, f)$ is not transversal to $W^{u}(x, f)$. Then $T_{x} W^{s}(x, f) \supset T_{x} W^{u}(x, f)$ and so $E=$ $T_{x} W^{s}(x, f) \cap T_{x} W^{u}(x, f)=T_{x} W^{u}(x, f)$. Take $\delta_{3}>0$ small enough, then there exist $\varepsilon^{\prime}>0$ and $0<\varepsilon<\varepsilon^{\prime}$ such that $W_{\varepsilon}^{u}(x, g) \subset \exp _{x}\left(E\left(\delta_{3}\right)\right) \subset W_{\varepsilon^{\prime}}^{s}(x, g)$ and $W_{2 \varepsilon^{\prime}}^{s}(x, g) \subset W^{s}(x, g)$. Since $g$ has POTP, there exists $\delta>0$ such that if $d(y, z) \leq \delta(y, z \in M)$ then $W_{\varepsilon}^{s}(y, g) \cap W_{\varepsilon}^{u}(z, g) \neq \varnothing$. Thus we have $W_{\varepsilon}^{s}(y, g)$ $\cap W_{\varepsilon}^{u}(x, g) \neq \varnothing$ for all $y \in B_{\delta}(x)$, and so $W_{\varepsilon}^{s}(y, g) \cap W_{\varepsilon^{\prime}}^{s}(x, g) \neq \varnothing$. Therefore $y \in W_{\varepsilon+\varepsilon^{\prime}}^{s}(x, g) \subset W_{2 \varepsilon^{\prime}}^{s}(x, g) \subset W^{s}(x, g)$, and so $B_{\delta}(x) \subset W^{s}(x, g)$. This contra$\operatorname{dicts} \operatorname{dim} W^{s}(x, f)=\operatorname{dim} W^{s}(x, g)=\operatorname{dim} M-1$.

\section{REFERENCES}

[1] N. Aoki, On homeomorphisms with pseudo orbit tracing property, Tokyo J. Math., 6 (1983), 329-334.

[2] - - The set of Axiom $A$ diffeomorphisms with no cycle, preprint.

[ 3 ] J. Franks, Necessary conditions for stability of diffeomorphisms, Trans. A.M.S., 158 (1971), 301-308.

[ 4 ] M. Hirsch and C. Pugh, Stable manifolds and hyperbolic sets, in Global Analysis, Proc. Sympos. Pure Math., A.M.S., 14 (1970), 133-163.

[5] R. Mañé, A proof of the $C^{1}$ stability conjecture, Publ. Math. I.H.E.S., 66 (1987), 161-210.

[6] A. Morimoto, Stochastically stable diffeomorphisms and Takens conjecture, Suriken Kokyuroku, 303 (1977), 8-24.

[ 7 ] Z. Nitecki, On semi-stability for diffeomorphisms, Invent. Math., 14 (1971), 83122.

[ 8 ] Z. Nitecki and M. Shub, Filtrations, decompositions and explosions, Amer. J. Math., 97 (1976), 1029-1047.

[ 9 ] J. Palis, On the $\mathrm{C}^{1} \Omega$-stability conjecture, Publ. Math. I.H.E.S., 66 (1987), 211215.

[10] M. Peixoto, Structural stability on two-dimensional manifolds, Topology, 1 (1962), 101-120.

[11] C. Robinson, $C^{r}$ structural stability implies Kupka-Smale, In: Dynamical Systems, edited by Peixoto. Academic Press (1973). 
[12] - Stability theorems and hyperbolicity in dynamical systems, Rocky Mountain J. Math., 7 (1977), 425-437.

[13] S. Smale, The $\Omega$-stability theorem, in Global Analysis, Proc. Sympos. Pure Math., A.M.S., 14 (1970), 289-297.

[14] P. Walters, Anosov diffeomorphisms are topologically stable, Topology, 9 (1970), 71-78.

[15] - On the pseudo-orbit tracing property and its relationship to stability, Springer L.N., No. 668 (1979), 231-244.

Department of Mathematics

Tokyo Metropolitan University

Minami-Ohsawa 1-1, Hachioji-shi

Tokyo 192-03

Japan 